\title{
RE: Role of rapid diagnostics for viral respiratory infections in antibiotic prescribing decision in the emergency department, by Li et al (2019)
}

\author{
Danielle A. Rankin MPH, $\mathrm{ClC}^{1,2}$ (1), Peter F. Rebeiro PhD, $\mathrm{MHS}^{3,4}$ (1) , Zaid Haddadin MD¹, Natasha B. Halasa MD, MPH ${ }^{1}$ \\ and Robert A. Greevy Jr, $\mathrm{PhD}^{4}$ \\ ${ }^{1}$ Department of Pediatrics and Institute for Global Health, Vanderbilt University Medical Center, Nashville, Tennessee, ${ }^{2}$ Vanderbilt University School of Medicine, \\ Nashville, Tennessee, ${ }^{3}$ Division of Infectious Diseases \& Division of Epidemiology, Department of Medicine, Vanderbilt University School of Medicine, Nashville, \\ Tennessee and ${ }^{4}$ Department of Biostatistics, Vanderbilt University Medical Center, Nashville, Tennessee
}

To the Editor-Li et al ${ }^{1}$ recently published a notable article evaluating the frequency of patients who had a viral respiratory infection (VRI) confirmed via rapid polymerase chain reaction (PCR) and were prescribed an antibiotic prior to discharge at 3 distinct emergency departments (EDs) within a single healthcare network. Antibiotic use is associated with adverse events and leads to antibiotic resistance. ${ }^{2,3}$ In the United States, $>2.8$ million antibiotic-resistance infections occur annually, which highlights the importance of research in appropriate antibiotic prescribing. ${ }^{3}$ In their study, Li et $\mathrm{al}^{1}$ conducted an exploratory analysis using multivariable logistic regression to evaluate the predictors influencing antibiotic prescriptions in patients with PCR-confirmed VRI prior to discharge from the emergency department. Their study reveals 3 notable predictors that we believe are likely to bear out as meaningful predictors. However, the study is also an excellent example of an underappreciated design challenge. We describe the challenge and provide some guidance to overcome it.

These authors selected covariates that had univariate associations with $P$ values $<.25$ and they then used a stepwise backward elimination to determine the final multivariable model. The initial covariates selected were age; immunocompromised status (ie, chronic kidney disease, diabetes mellitus, human immunodeficiency virus, or an active malignancy); receipt of antibiotics within 7 days prior to their ED visit; duration of symptoms; hemoglobin levels; abnormal chest $\mathrm{x}$-ray results; and discharge diagnoses of influenza, upper respiratory tract infection, pneumonia, and otitis media. ${ }^{1}$ In table 3 of this article, the final model reported effect estimates for the following covariates: receipt of antibiotics within 7 days prior to the ED visit, immunocompromised state, abnormal chest $\mathrm{x}$-ray results, and discharge diagnosis of pneumonia. ${ }^{1}$ Readers should use caution when interpreting results like these because they may be biased due to what is known as the "Table 2 Fallacy." The "Table 2 Fallacy" is when effect estimates for multiple exposures and their confounders are estimated from the same statistical model, results that are often presented in an article's "Table 2." 5,6 Specifically, the covariates included in the final model of this study are secondary exposures of interest and may be serving as confounders to VRIs and/or antibiotic prescriptions. Therefore, the interpretation of the effect estimates across

Author for correspondence: Danielle A. Rankin, E-mail: Danielle.A.Rankin@ vanderbilt.edu

Cite this article: Rankin DA, et al. (2020). RE: Role of rapid diagnostics for viral respiratory infections in antibiotic prescribing decision in the emergency department, by $\mathrm{Li}$ et al (2019). Infection Control \& Hospital Epidemiology, 41: 991-992, https:// doi.org/10.1017/ice.2020.123

(C) 2020 by The Society for Healthcare Epidemiology of America. All rights reserved. the modeled covariates may change if any of those covariates themselves are confounded by other covariates that are not included in the model. This can happen even when the primary study exposure is not confounded.

To illustrate, we have calculated crude odds ratios for the covariates included in the final model. Based on table 3 of Li et al, ${ }^{1}$ VRI serves as the primary exposure and the prescription of antibiotics is the outcome. In this model, all of the covariates are adjusted for the exposure of viral respiratory infection. However, when creating the final model through the backward stepwise method, the potential confounder(s) influencing the other covariates in the final model may have been dropped. For instance, the variable immunocompromised status is likely influenced by the variable age. Because the final model does not adjust for the confounder age, the results reported do not provide an unbiased effect estimate for immunocompromised status. Additionally, the other covariates reported in table 3 may have confounders that were not conditioned on in the final model, thus misrepresenting the true, unbiased effect estimate. With respect to the criteria the authors used to classify immunocompromised status (ie, chronic kidney disease, diabetes mellitus, human immunodeficiency virus, and an active malignancy), with the exception of HIV, these conditions are directly affected by age. The odds ratio (OR) reported for immunocompromised status was 3.51 (95\% confidence interval [CI], 1.44-8.81). However, when we calculated the crude odds ratio of immunocompromised status, the ratio decreased to 1.72 (95\% CI, 0.91-3.16) (Table 1). If age were adjusted for, we could potentially see a different effect estimate altogether, even if age was not directly associated with the outcome.

With the increase of antibiotic resistance, research evaluating components that contribute to antibiotic prescribing practices is vital to assist in formulating interventions for limiting inappropriate antibiotic use. Overall, we believe Li et al provide important contributions toward understanding antibiotic prescribing practices in the ED. To circumvent the misinterpretation of multiple effect estimates in future research, we suggest using multiple models that are tailored to generate unbiased effect estimates for the exposure(s) of interest. ${ }^{4,5}$ In addition, we suggest explicitly specifying between the unadjusted and adjusted effect measures from a single logistic regression model in the footnotes of the table. ${ }^{4,5}$ Conditioning on covariates should be firstly based on a priori knowledge of how the variables influence the primary exposure and/or outcome; and secondly on the observed effect sizes. A statistically nonsignificant covariate may still meaningfully 
Table 1. Adjusted Versus Unadjusted Effect Estimates, Highlighting the "Table 2 Fallacy"

\begin{tabular}{lcc}
\hline Covariate & $\begin{array}{c}\text { Adjusted Odds Ratio } \\
(95 \% \mathrm{Cl})\end{array}$ & $\begin{array}{c}\text { Crude Unadjusted Odds } \\
\text { Ratio }(95 \% \mathrm{CI})^{\mathrm{a}}\end{array}$ \\
\hline $\begin{array}{l}\text { Antibiotics in the } \\
\text { previous 7 days }\end{array}$ & $7.65(3.13-19.42)$ & $3.567(1.79-6.97)$ \\
\hline Immunocompromised & $3.51(1.44-8.81)$ & $1.716(0.910-3.16)$ \\
\hline Abnormal chest x-ray & $2.54(1.07-5.98)$ & $5.174(2.99-9.42)$ \\
\hline $\begin{array}{l}\text { Discharge diagnosis of } \\
\text { pneumonia }\end{array}$ & $63.3(15.1-445)$ & $40.55(13.20-167.85)$ \\
\hline
\end{tabular}

Note. $\mathrm{Cl}$, confidence interval.

${ }^{a}$ Crude unadjusted odds ratios were calculated in R studio software with the EpiTools package (R Foundation for Statistical Computing, Vienna, Austria).

reduce bias, and a statistically significant one with a small effect size may have trivial impact on bias.

Acknowledgments. None.

Financial support. No financial support was provided relevant to this article.
Conflicts of interest. All authors report no conflicts of interest relevant to this article.

\section{References}

1. Li J, Kang-Birken SL, Matthews SK, Kenner CE, Fitzgibbons LN. Role of rapid diagnostics for viral respiratory infections in antibiotic prescribing decision in the emergency department. Infect Control Hosp Epidemiol 2019;40:974-978.

2. Fleming-Dutra KE, Hersh AL, Shapiro DJ, et al. Prevalence of inappropriate antibiotic prescriptions among US ambulatory care visits, 2010-2011. JAMA 2016;315:1864-1873.

3. Antibiotic/antimicrobial resistance biggest threats and data. Centers for Disease Control and Prevention website. https://www.cdc.gov/drugresistance/ biggest-threats.html. Published 2019. Accessed December 1, 2019.

4. Westreich D, Greenland S. The table 2 fallacy: presenting and interpreting confounder and modifier coefficients. Am J Epidemiol 2013;177: 292-298.

5. Bandoli G, Palmsten K, Chambers CD, Jelliffe-Pawlowski L, Baer RJ, Thomspon CA. Revisiting the table 2 fallacy: a motivating example examining preeclampsia and preterm birth. Paediatr Perinat Epidemiol 2018; 32:390-397.

\title{
Role of telehealth in outbreaks-Where the classical healthcare systems fail
}

\author{
Ata Mahmoodpoor MD, FCCM ${ }^{1}$ (1), Mohammad Amin Akbarzadeh ${ }^{2}$ (ㄷ), Sarvin Sanaie MD, $\mathrm{PhD}^{3}$ (1) and \\ Mohammad-Salar Hosseini ${ }^{4}$ \\ ${ }^{1}$ Department of Anesthesiology and Critical Care Medicine, Faculty of Medicine, Tabriz University of Medical Sciences, Tabriz, Iran, ${ }^{2}$ Student Research \\ Committee, Tabriz University of Medical Sciences, Tabriz, Iran, ${ }^{3}$ Aging Research Institute, Tabriz University of Medical Sciences, Tabriz, Iran and ${ }^{4}$ Research \\ Center for Evidence-Based Medicine, Tabriz University of Medical Sciences, Tabriz, Iran
}

To the Editor-Outbreaks impose massive burdens on healthcare systems. For example, $>510$ deaths were reported among the healthcare workers (HCWs) during the last Ebola outbreak, and by February 24, 2020, >3,300 HCWs had been infected with SARS-Cov-2 in China alone. ${ }^{1,2}$ This issue represents the essence of outbreaks - a mass of patients who require prompt medical care. Under these conditions, the classical healthcare system cannot manage the large number of COVID-19 patients immediately and effectively. ${ }^{3}$

Telehealth provides vital services through the application of information and communication technologies for each stage of managing diseases, research, and continuing education. ${ }^{4}$ With telecommunication, visits are more economical and preserve government, community, and family resources, which are already limited during global outbreaks. It can be used to provide rapid diagnosis, and it enables caregivers to act quickly. ${ }^{5}$ With the appropriate utilization of telecommunication, it is possible bring access to medicine and concurrent therapy in hard-to-reach regions and

Author for correspondence: Mohammad-Salar Hosseini, E-mail: hosseini.msalar@ gmail.com

Cite this article: Mahmoodpoor A, et al. (2020). Role of telehealth in outbreaksWhere the classical healthcare systems fail. Infection Control \& Hospital Epidemiology, 41: 992-994, https://doi.org/10.1017/ice.2020.120 communities, facilitating direct-to-patient or specialty consultation services from a distance, which is cost-effective and improves the efficacy of follow-up. ${ }^{6}$

The benefits of implementing telehealth in outbreaks comprise 8 main areas of focus:

1. The available admission capacity and the number of HCWs are limited, and the hospital infrastructure may not be adequate to serve all patients. Figure 1 presents an optimum model of telemedicine that could aid medical and social management during an outbreak.

2. The more patients referred to healthcare facilities, the more HCWs are at risk of being infected. Also, most referrals are unnecessary and merely increase the load on the healthcare system. Furthermore, people referred to hospitals are at an incredibly higher risk of infection. More than $40 \%$ of the infections are assumed to be hospital related, though they should be quite simple to prevent (Fig. 1). ${ }^{7}$

3. In affected regions, most healthcare facilities are dedicated to the management of COVID-19 patients. Therefore, noncritical patients (like patients with chronic and metabolic disorders such as hypertension, diabetes, and hyperlipidemia) have a lower priority. As a result of self-isolating, these people, mostly the older adults, cannot attend the healthcare units. A

(c) 2020 by The Society for Healthcare Epidemiology of America. All rights reserved. This is an Open Access article, distributed under the terms of the Creative Commons Attribution licence (http://creativecommons.org/licenses/by/4.0/), which permits unrestricted re-use, distribution, and reproduction in any medium, provided the original work is properly cited. 